Progress in

Aerospace and

Aviation

Technology

\title{
Sudden Obstacle Appearance Detection by Analyzing Flow Field Vector for Small-Sized UAV
}

\author{
Muhammad Faiz Ramli ${ }^{1}$, Syariful Syafiq Shamsudin'², Mohd Fauzi Yaakub ${ }^{1 *}$
}

${ }^{1}$ Aircraft System and Design Research, Faculty of Mechanical and Manufacturing Engineering, Universiti Tun Hussein Onn Malaysia, Batu Pahat, 86400, Johor, MALAYSIA

${ }^{2}$ Research Center of Unmanned Vehicle, Faculty of Mechanical and Manufacturing Engineering, Universiti Tun Hussein Onn Malaysia, Batu Pahat, 86400, Johor, MALAYSIA

*Corresponding Author

DOI: https://doi.org/10.30880/paat.2021.01.01.004

Received 24 September 2021; Accepted 11 November 2021; Available online 21 December 2021

\begin{abstract}
Achieving a reliable obstacle detection and avoidance system that can provide an effective safe avoidance path for small unmanned aerial vehicle (UAV) is very challenging due to its physical size and weight constraints. Prior works tend to employ the vision based-sensor as the main detection sensor but resulting to high dependency on texture appearance while not having a distance sensing capabilities. The previous system only focused on the detection of the static frontal obstacle without observing the environment which may have moving obstacles. On the other hand, most of the wide spectrum range sensors are heavy and expensive hence not suitable for small UAV. In this work, integration of different based sensors was proposed for a small UAV in detecting unpredictable obstacle appearance situation. The detection of the obstacle is accomplished by analysing the flow field vectors in the image frames sequence. The proposed system was evaluated by conducting the experiments in a real environment which consisted of different configuration of the obstacles. The results from the experiment show that the success rate for detecting unpredictable obstacle appearance is high which is $70 \%$ and above. Even though some of the introduced obstacles are considered to have poor texture appearances on their surface, the proposed obstacle detection system was still able to detect the correct appearance movement of the obstacles by detecting the edges.
\end{abstract}

Keywords: Multi sensor integration, flow field vector, small sized uav, safe avoidance path, sudden obstacle

\section{Introduction}

Hypothetically, the UAV platform has great potential to perform numerous tasks such as monitoring the environment [1], massive building and structure inspection [2, 3], search and rescue activities [4, 5, 6] and others. Most of these tasks require the UAV to achieve a higher level of autonomy in its embedded system. One of the tasks in the autonomous system for the UAV is the operation to identify the appearance of any obstacles that are being introduced to the UAV and ultimately create a manoeuvre action plan. The obstacle detection and avoidance operation can be very challenging to the UAV platform, especially for a small-sized UAV. This is due to the payload capacity and physical size constraints of the UAV. Typically, the obstacle detection system for UAV depends on the type of sensors being installed onboard the UAV, which is either vision-based sensors or range-based sensors. Selecting the proper sensors to be placed onboard the UAV plays a critical role in the system operation, where each of the aforementioned techniques has its own advantages and disadvantages. For example, the vision-based sensor method can provide rich information regarding the bearing of the detected obstacles in the operating environment. However, the distance from the UAV to obstacles are poorly recognised and estimated. Besides, the detection will heavily rely on the texture appearance of the obstacle. Conversely, 
range-based sensor is excellent in determining the distance value of the detected obstacle, but there is a lack of information about the location of the detected obstacle in the surrounding environment.

Since most of the commercial UAVs at present are small in size, the development of the obstacle detection system becomes more complicated due to the mentioned constraints. As a result, the researchers need to find a balanced line between the performance of the system and the constraints by the UAV platform. UAV needs a robust obstacle detection system that can determine trusted safe path avoidance regardless of texture appearance, obstacles sizes and appearance of the obstacles introduced in the environment. This matter is especially vital if the appearance of the obstacles is unpredictable and moving dynamically in front of the UAV. On top of that, the system must perceive a robust distance value to obstacles, so that, warning for collision and decision for avoidance can be made. Although it may be possible for a larger UAV, it is still difficult for small UAV to achieve. Therefore, in this paper, we present a method to meet these needs and enhance the detection capability by integrating multi-sensors which are camera sensor and simple Lidar-based sensors into the system. We use LIDAR sensor as initial detection in term of detecting distances and queue for activating the camera. To detect the movement and direction of the moving obstacles in front of the UAV, we use the optical flow method and analyze the direction of the flow field vector in the image frames sequence.

The focus of the research can be observed through these questions: First, would it be possible to integrate the rangebased sensor and vision-based sensor together into one detection system to enhance the performance of the detection for small-sized UAV? Secondly, how to detect and avoid the obstacles that is appear suddenly in front of the UAV? Finally, will the proposed detection system able to detect and recognize the appearance of poor textured obstacle or textureless obstacle in the environment?

The rest of this paper is organized as follows. Section 2 present the method from the previous work. The proposed obstacle detection system is decribed in Section 3. After that, Section 4 describe the implementation and experimental setup. Followed by result and discussion in Section 5. Finally, Section 6 is dedicated to the conclusion and future work

\section{Related Works}

Constraints that are mentioned above are the ultimate factors for the researcher to choose the vision-based sensor as the source of the environmental information for the obstacle detection operation. Most commercial UAVs are already equipped with the vision-based sensor, which is the monocular camera sensor. By using the proper vision algorithms, the appearance of the obstacle in the operating environment can be detected [7]. For example, [8] and [9] have employed the optical flow method produced by the image frames sequence to avoid any obstacle that can pose a catastrophic to the UAV platform. However, this method is only reliable and effective for centring the UAV in the operating environment and will not be able to detect unpredictable appearance of the obstacles. Besides, the optical flow method has poor ability in detecting the appearance of the frontal obstacle $[10,11]$.

[12] uses edge detection to detect the free zones in flying environment. However, this method will be less reliable if the environment is fully textured. Another example is the feature size expansion $[10,13]$. This method suffers from creating a trusted safe avoidance path after encountering the detected obstacle in the operating environment. It is unable to detect the free region available from the observed operating environment, whereby the safe avoidance path is thus assumed by the tolerance extension from the detected obstacle feature points. As a consequence, the path for the avoidance manoeuvre is not reliable considering any encountered obstacles will have random textures and features.

In most cases, the vision algorithms available will depend a lot on the texture of the obstacles; if the obstacle surface is texture-less, it will not be able to execute the detection process. Moreover, previous vision obstacle detection and avoidance systems only focus on the detection of the obstacle that is situated ahead and directly in front of the UAV [14, $11,10]$. In relation to that, the UAV will be exposed to collision risk during mission operation considering the vision sensor does not contain any distance sensing capabilities. On top of that, the detection for sudden appearance of the obstacle is still a problem for the detection system. Considering that the vision-based obstacle detection system will highly depends on the computation of the vision algorithm in each consecutive captured image frames, the possibility to have latency on the computation in real time will be high [15]. In addition, the judgement towards the obstacle with respect to its estimated distance and bearing will also be negatively affected (real time effect). In order to work with depth estimation of the environment, researchers tend to employ the stereo vision sensor to relatively estimate the distance of the observed environment from the UAV, which includes the obstacles [16, 17]. However, the major problem for the stereo vision sensor is the length constraints of the baseline for both of the attached camera sensors, which in turn can decrease the accuracy of the estimation. In addition, the weight and size of the camera are also considered as problems for the stereo vision sensor system.

Apart from the vision-based sensor, the range-based sensor $[18,19,20]$ has also been explored by the researchers to be used in the obstacle detection system. Although the range-based sensor is very reliable in detecting the appearance of the obstacles in predictable and unpredictable situation, most of the reliable range-based sensors (e.g. SICK, Hokuyo, Velodyne Puck and etc.) are heavy, massive, and very expensive to be practically used in a small-sized commercial UAV. Our approach uses integration of different based-sensor to enhance the capability of the system in detecting the sudden appearance of the obstacle in front of the UAV when compared to single sensor obstacle detection system. Our work analyzed the flow field vector direction derived from the optical flow method to detect the appearance of the obstacle. 


\section{Framework of Proposed Obstacle Detection System}

The framework of the proposed system contains three modules, which are the sensing module, obstacle detection module, and avoidance module as illustrated in Figure 1. In sensing module, there are 2 sensors used in the system which are LIDAR and monocular camera sensor. Apart from detecting the distance of the obstacle from the UAV platform, the LIDAR sensor will also acts as the safety device for the proposed obstacle detection and avoidance system by maintaining the safe distance from any objects. In addition, the capability of the LIDAR sensor to scan the environment is needed during the avoidance manoeuvre to ensure the safety of the avoidance path. The distance detection procedure will be applied to both situations which are predictable and unpredictable obstacle appearance situations. In this paper, only unpredictable obstacle appearance situation is discussed (obstacle detection module).

For the vision-based sensor, the image frames captured by the camera sensor are used to detect the appearance or motion of the obstacle in the environment, so that, the distance and sudden movement of the obstacles can be observed. In obstacle detection module, the detection is achieved by analyzing the direction of the flow field vector derived from the optical flow method. After the obstacles in the image frame are identified, the free regions are developed to produce the path for safe avoidance known as the safe avoidance path. Finally, once the safe avoidance path is identified, the avoidance manoeuvre will be executed.

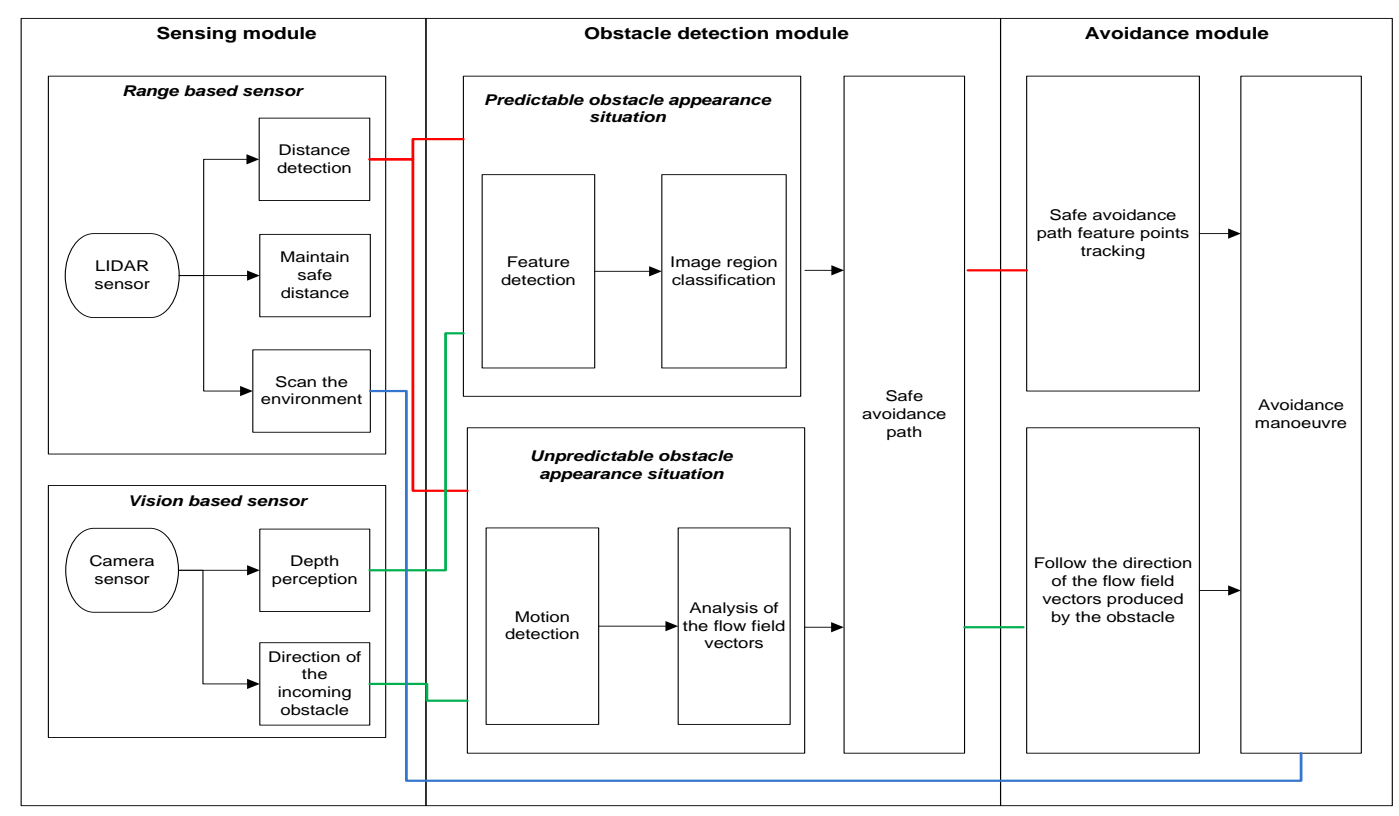

Fig. 1 - Framework of the proposed obstacle detection system

\subsection{Sudden Obstacle Appearance Detection Operation}

The detection technique in the unpredictable obstacle appearance situation will be based on the observation of the flow field vector generated from the optical flow. The general process of the sudden obstacle appearance detection operation is shown in Figure 2. As can be observed from the figure, the sudden obstacle appearance detection technique depends on analysing the direction of the flow field vectors generated by the optical flow method (Lukas-Kanade algorithm). [21] and [22] stated that the Lucas-Kanade method is much faster in computation than the Horn-Schunck method.

In order to generate or detect the presence of the flow field vectors in the surrounding environment, the optical flow method will be applied to the sequence of image frames captured by the camera sensor. In order to reduce the computational time and complexity of the proposed obstacle detection and avoidance system, it is favourable to activate the sudden obstacle appearance detection (optical flow detection) only after the obstacle is detected below than $150 \mathrm{~cm}$ range (distance of $150 \mathrm{~cm}$ is decided in predictable obstacle appearance situation). Hence, instead of computing the optical flow for every image frame sequence during the UAV operation, the optical flow will only be computed on the detected obstacle image frame sequence which is the image frame sequence after the obstacle is detected.

However, it can become dangerous to the UAV when the detected obstacle stops from moving right after it was detected by the LIDAR sensor (see Figure 3(a)). If that scenario happens, the computed optical flow will most likely become unreliable to the proposed obstacle detection and avoidance system. This is because the direction of the computed optical flow can easily be swayed by the movement of the camera sensor from the UAV even though the obstacle is not moving or at rest. The proposed obstacle detection system must take into account this scenario to become robust and reliable. Thus, in the proposed obstacle detection system, the optical flow detector will be an active detection system, which means it will operate at all times during the UAV operation. The sudden obstacle appearance detection system will 
use the image frame sequence before the detected obstacle image frame to compute the optical flow as illustrated in Figure 3(b). It is considerably more reliable and safer for the UAV during the operation.

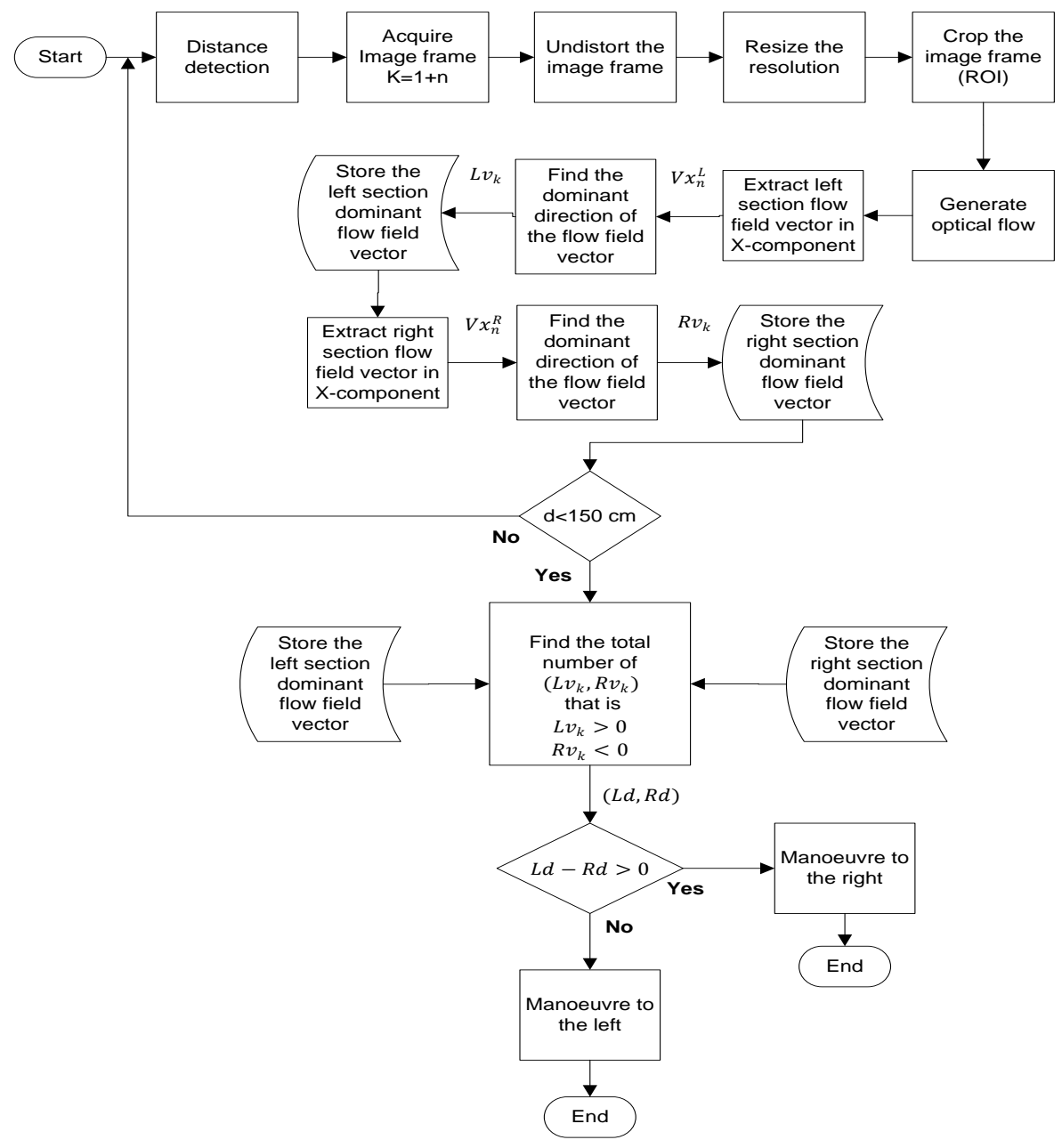

Fig. 2 - Flow of sudden obstacle detection operation
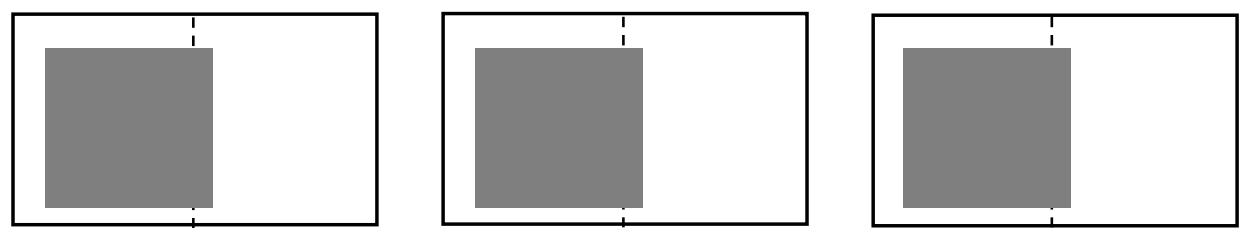

(a)
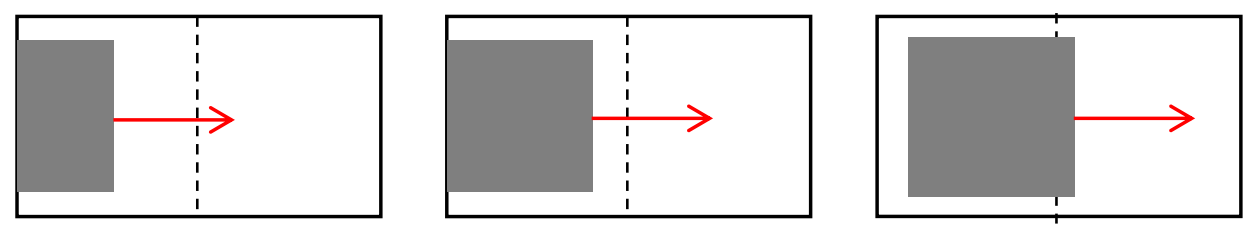

(b)

Fig. 3 - (a) The obstacle stop from moving right after the detection is initiated ; (b) The detection is activated before the obstacle is detected by the LIDAR Lite v3. Red arrow indicates the movement of the obstacle throughout the image frame sequence

Since the optical flow detector will operate at all time during the UAV operation, the computational time and complexity will be relatively high due to the computation of optical flow on every image frame sequence during the UAV operations. Consequently, this high computational time and complexity can undeniably jeopardise the obstacle detection 
system in a real-time application. In an effort to reduce the computational time and complexity, the image frame configuration will be configured to suit the real-time application. The image frame resolution in this particular situation will be reduced to $320 \times 240$. In addition, the optical flow detector only operates in specific ROI of the image frame, which is the centre area of the image frame. As seen in Figure 4, the ROI dimension consists of 200 pixels in width and 240 pixels in height.

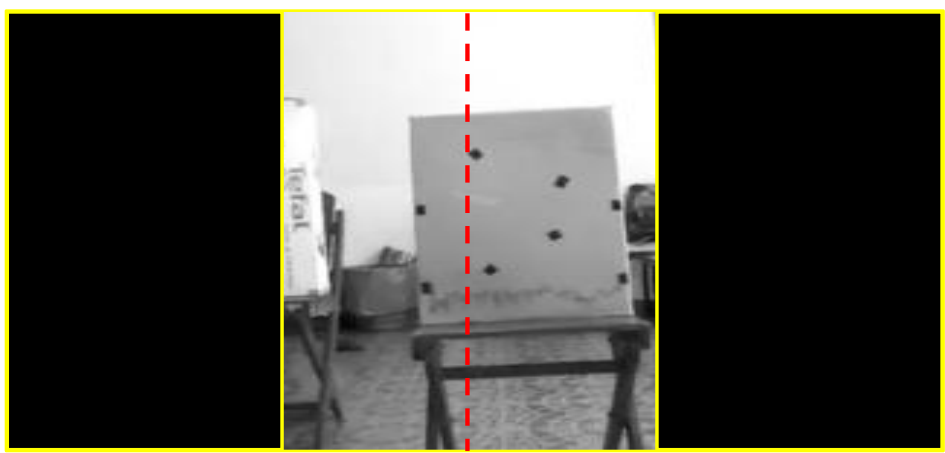

Fig. 4 - Selected ROI in the image frame for sudden appearance of the obstacle situation

Unlike previous researchers [23, 8, 24], they employ the optical flow detector in their obstacle detection system to measure the depth of the surrounding environment, therefore, it is very critical to measure the optical flow all around the image frame, so that the overall depth of the surrounding environment can be interpreted. However, the depth of the environment or distance from the obstacle can easily be determined by the LIDAR sensor in the proposed obstacle detection and avoidance system. Therefore, the information about the distance of the obstacle to the UAV would not become an issue for the proposed obstacle detection system. The only problem for the proposed obstacle detection system is to determine which direction the obstacle will appear in front of the UAV. This direction information can be achieved only by observing the optical flow in the stated ROI without computing the optical flow in the whole area of the image frame. If the area of the ROI is increased, the computational time and complexity will be high. Meanwhile, if the area of the ROI is decreased, the direction information from the optical flow might be lost. On this account, the selected position and area of the ROI is assumed to be sufficient for the computation by the proposed detection system.

The direction information by the optical flow is vital to the proposed obstacle detection system because this information enables the proposed obstacle detection system to decide which path the UAV should take to avoid the detected obstacle. Rather than only be able to detect and know the distance of the obstacle from the UAV, it is important to acknowledge the direction of the incoming obstacle as well. For example, if the obstacle suddenly appears and stops in front of the UAV and it is coming from the left side, then, it is safer that the proposed detection system creates the safe avoidance path away from the incoming obstacles, which in this case, is on right side of the UAV (see Figure 5). On top of that, the proposed detection system does not have the ability to determine the physical configuration of the incoming obstacle and thus, it will be dangerous for the UAV to go to the direction of the incoming obstacle for avoidance.
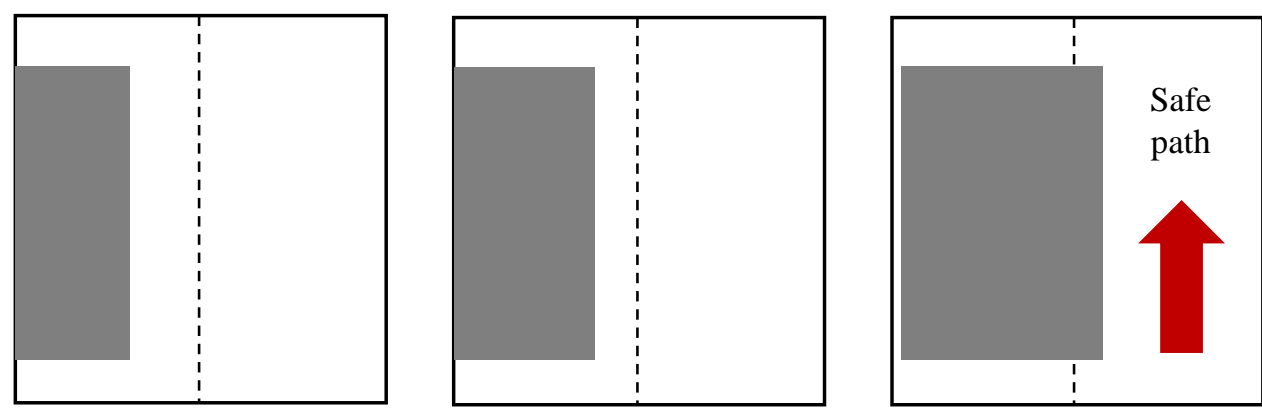

Fig. 5 - Safe avoidance path when encountering the sudden appearance obstacle situation

In general, when the UAV (camera sensor) moves forward through the environment, the optical flow vectors will displace away from the centreline of the UAV as illustrated in Figure 6. If an object moves inside this environment and towards the UAV position, the optical flow generated will be similar to the movement of the object. As a result, there are two focus of expansions (FOE) in the image frame sequence recorded by the camera sensor. FOE refers to a single point where all of the flow fields appear to be coming from. If there are multiple FOEs in the image frame sequence, which is 
similar to a real situation when the obstacle suddenly appear in front of the moving UAV, the optical flows detected need to be analysed properly because it contains a mixture of flow fields between the detected obstacles and background.

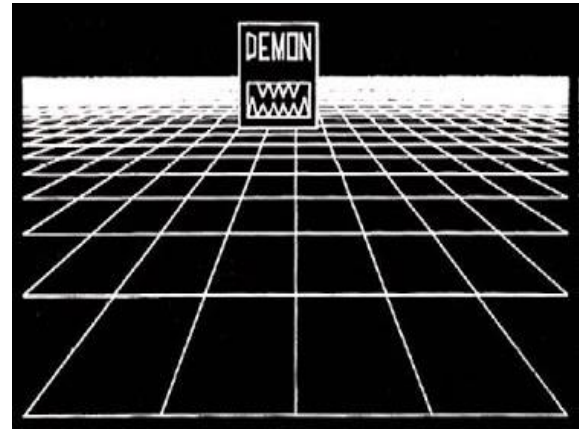

(a)

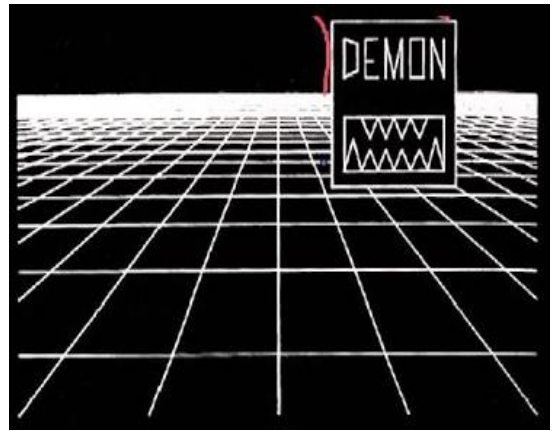

(b)

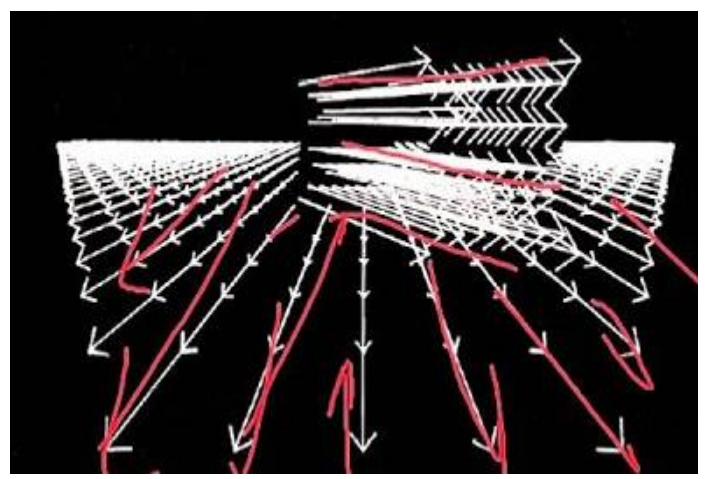

(c)

Fig. 6 - (a) UAV moves forward with the object is static in the environment; (b) UAV moves forward with the object moves towards the UAV; (c) Observed FOE

The ROI on the centre area of the image frame will be further divided into two sections, which are referred to the left and right sides of the UAV as illustrated in Figure 7. The direction of the optical flows in the $\mathrm{x}$-direction $\left(V x_{n}^{L}, V x_{n}^{R}\right)$ within these sections able to provide the direction of the incoming obstacle to the proposed detection system. $V x_{n}^{L}$ and $V x_{n}^{R}$ refer to the flow field vectors in the left section and right section of the image frame respectively (within the ROI). When the obstacle is coming from the right side of the UAV, the generated optical flows will have a negative flow field vector in x-direction $V x_{n}^{R}$ and vice versa, when the obstacle is coming from the left side of the UAV. Once the obstacle suddenly appears in front of the UAV, the proposed obstacle detection system will identify the dominant direction of the flow field vector flows from the previous image frame sequence on both left section $L v_{K}$ and right section $R v_{K}$.

Since the requirement to compute the motion of the object or optical flow requires at least two image frames sequence, the proposed obstacle detection system compute the optical flow from five of the previous image frame sequence in order to become a conservative detection system. For example, during the UAV operation or mission, when an obstacle suddenly appears in front of the UAV and it is coming from the right side of the UAV, the right section of the ROI will contain a higher total number of negative flow field vector in x-direction for previous five image frames $R d$ than the total number of positive flow field vector in $\mathrm{x}$-direction for left section $L d$ in the previous five image frames.

Therefore, the proposed obstacle detection system is able to recognize which sections contain the optical flow that is out of the normal or opposite from the flow field theory. In this case, the right section contains the flow field vector direction $V x_{n}^{R}$ that is opposite from the flow field theory and thus, the proposed obstacle detection system will perceive that there is an obstacle (sudden appearance) and it is coming from the right side of the UAV. Once the direction of the sudden obstacle $D s$ is known, the UAV will execute the avoidance action by manoeuvring away from the detected sudden obstacle. 


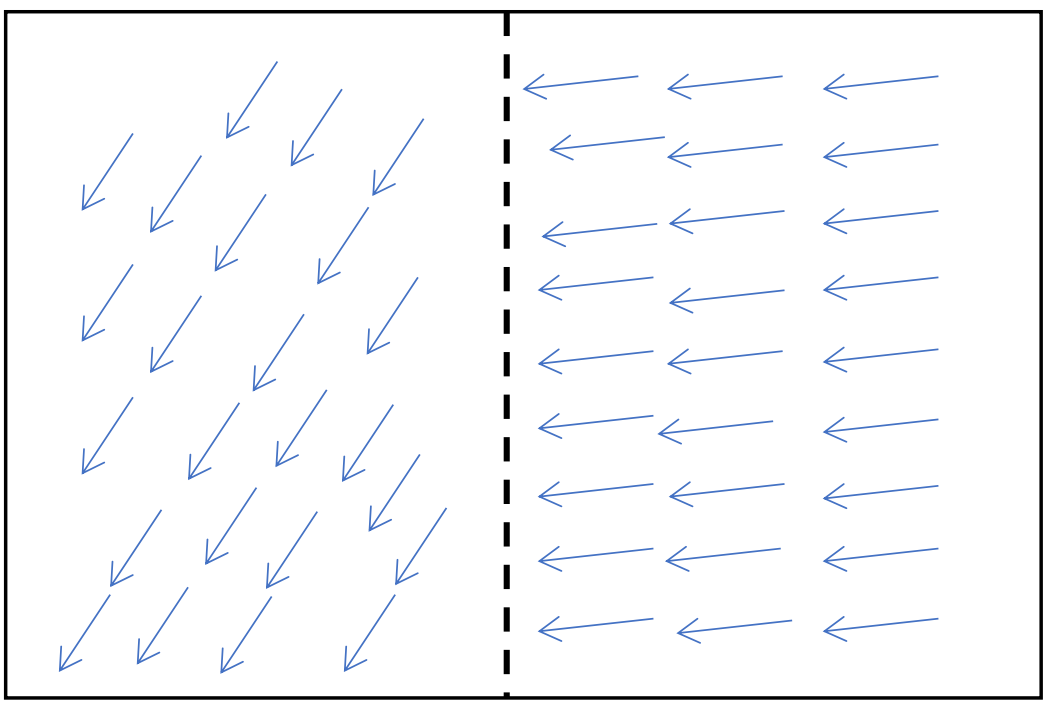

Fig. 7 - Direction of the optical flows from the selected ROI

\section{Implementation and Experimental Setup}

The platform used in the experimentation is the AR drone 2.0 elite edition, a low-cost UAV built by the company Parrot. This UAV was selected because it is a low-cost small commercial UAV available in the market and also has stable flying properties. Camera sensors used is the built-in UAV camera with 1280x720 resolution and horizontal field of view is 62 degrees. However, we use a lower resolution (320x240) to increase the computation speed of the algorithm. For range sensors, LIDAR Lite v3 is selected. It is selected because the measured range is very accurate and high as compared to other sensors like Infrared and Ultrasonic sensors. Also, it is considered low-cost and small in sizes when compare with other wide scanning Lidar. Arduino Nano v3.0 ATMEGA328 is used to read and connect Lidar sensor to the system (see Figure 8). In this research, the primary tool or software that we used for analysis and calculation is Matlab 2017a. The software contains a computer vision system toolbox which makes it possible to use as tool development for our obstacle detection and avoidance system. Our entire algorithm processed on a ground laptop which is quad-core Intel i7 running Windows operating system. Communication and data transfer between UAV including LIDAR sensor and ground laptop are done by XBee radio module.

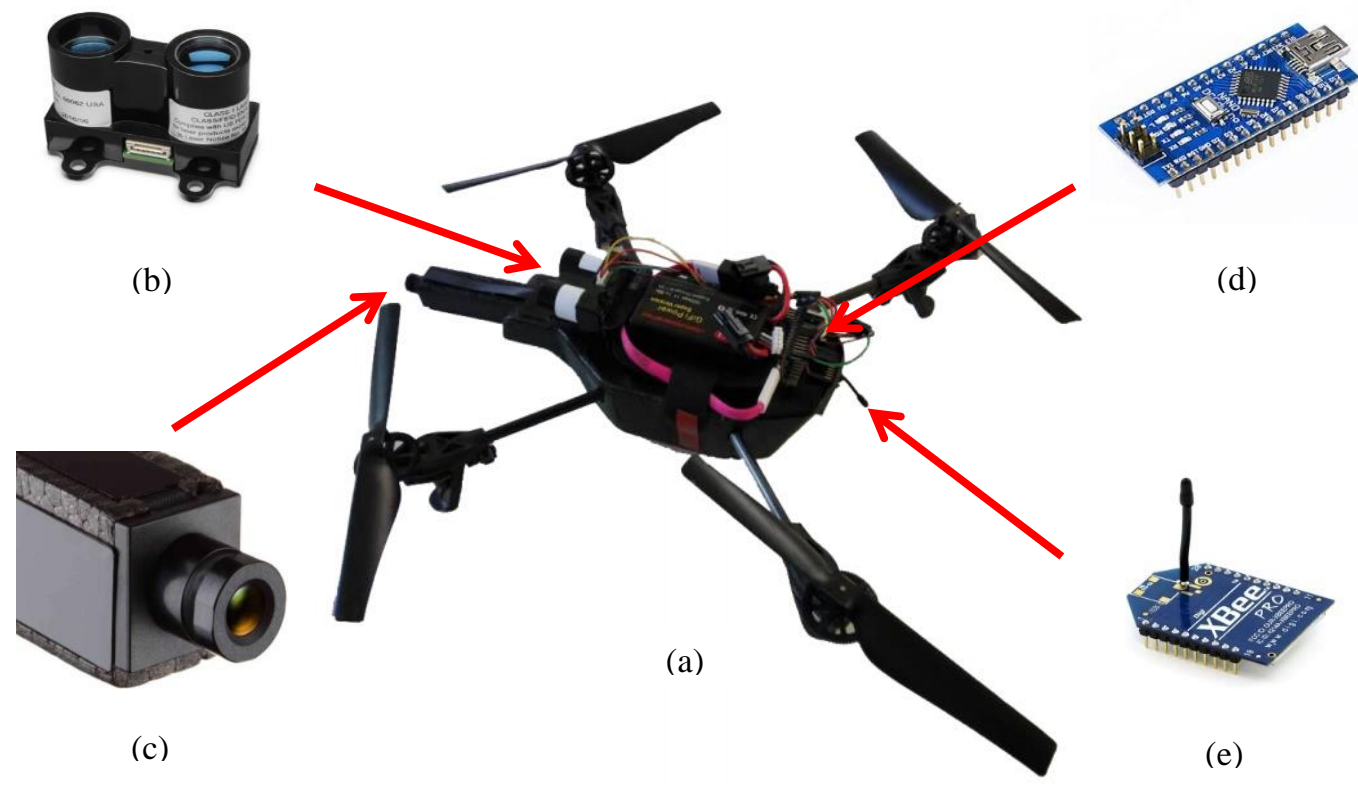

Fig. 8 - UAV platform configuration. (a) AR Drone 2.0; (b) Lidar Lite v3; (c) Camera sensor; (d) Arduino Nano; (e) Xbee Pro s1 
In order to evaluate the performance and capability of the proposed system, a number of obstacle detection and avoidance experiments with different configuration of the obstacles have been carried out. All of the algorithm processes and computations will be carried out by the external ground station (Intel i7-2670QM processor) with the help of Software Development Kit V.1.1. The external software selected for the video streaming channel from the UAV are the FFmpeg and ManyCam. The obstacles used in the experiments are shown in Figure 9 and note that these introduced obstacles vary in sizes and textures.

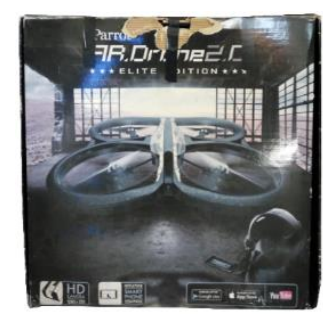

(a)

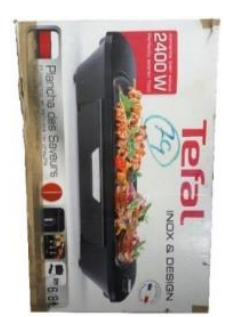

(b)

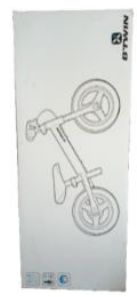

(c)

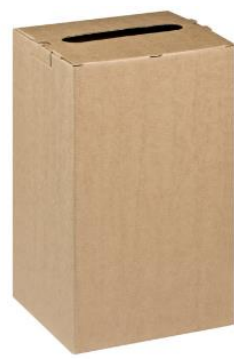

(d)

Fig. 9 - Obstacles used in the experiment. (a) Obstacle $1($ good texture); (b) Obstacle 2 (good texture); (c) Obstacle 3 (poor texture); (d) Obstacle 4 (texture-less)

The introduced obstacles will be brought directly in front of the UAV from either side (left side or right side) as depicted in Figure 10. Once the obstacle is detected, the proposed obstacle detection system will directly enter into the unexpected situation mode in which the sudden obstacle appearance detection operation will be executed (see Figure 2). Each of the experiment scenarios will be performed multiple times in real time for validation purposes.

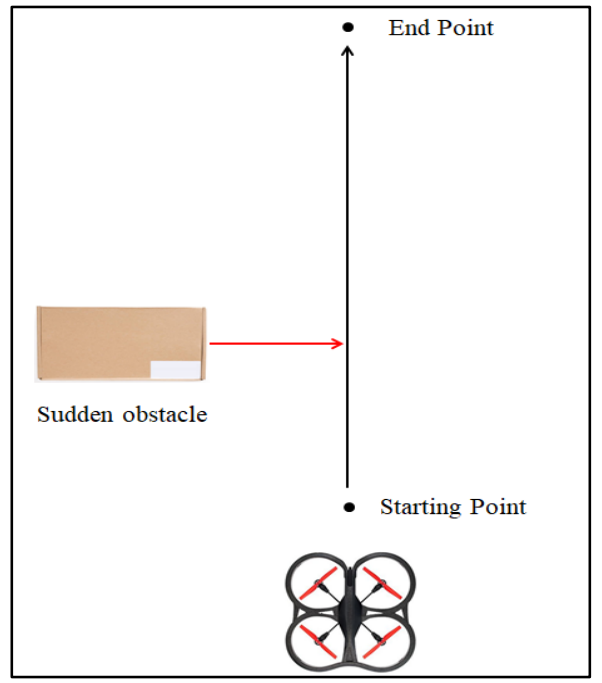

Fig. 10 - Experiment for unpredictable obstacle appearance situation

\section{Result and Discussion}

In this section, the results of the experiment for unpredictable obstacle appearance situation (sudden appearance of the obstacle) will be presented and discussed. The result of the real-time experiments for the second scenario is presented in Table 1. In this experiment, the success and failure of each run are measured by the ability of the proposed obstacle detection system to produce the desired manoeuvre decision for the UAV platform, which is manoeuvring away from the direction of the incoming introduced obstacles. The reason for this particular desired manoeuvre decision has already been discussed earlier in Section 3.1. As can be observed from the table, all of the obstacle cases have a relatively high success rate, which is about $70 \%-90 \%$. Regardless of the texture surface of the obstacles, the proposed obstacle detection system is able to produce reliable desirable manoeuvre decisions for the UAV platform. Even though some of the introduced obstacles are considered to have poor texture appearances on their surface, the proposed obstacle detection system was still able to detect the correct appearance movement of the obstacles. 
Table 1 - Result of experiment

\begin{tabular}{ccccc}
\hline Obstacle case & Total & Success & Failure & $\begin{array}{c}\text { Success } \\
\text { rate, } \%\end{array}$ \\
\hline 1 & 10 & 7 & 3 & 70 \\
2 & 10 & 8 & 2 & 80 \\
3 & 10 & 7 & 3 & 70 \\
4 & 10 & 9 & 1 & 90 \\
\hline
\end{tabular}

It is evident in the obstacle Case 4 (texture-less obstacle), where the success rate is the highest compared to the other obstacle cases. The high success rate for the obstacle Case 4 is due to the nature of the texture appearance itself. When the texture-less obstacle appears directly in front of the UAV from either side of the UAV as illustrated in Figure 11(a), a huge number of the flow field vectors will be generated on the edges of the obstacle rather than the surface itself. Therefore, the proposed obstacle detection system system will still be able to deduce the direction of the incoming obstacle from the flow field vectors located on the edges of the obstacle. In this case, a large number of positive flow field vectors are detected in the negative flow field region, which is the left section of the image frame. This behaviour indicates that there is an obstacle coming in front of the UAV and it is approaching the UAV from the left. As a result, the proposed obstacle detection system will create a safe avoidance path by manoeuvring the UAV to the left (moving away) until the UAV is safe and clear from the obstacle. Similar to the obstacle Case 4, the flow field vector detected for other obstacle cases will be a lot on the edges as well as on the surface of the obstacles (see Figure 11(b)). The flow field vectors detected on the surface depend a lot on the texture appearance of the obstacle. Ultimately, by observing the overall result of the experiments, it is safe to assume that the texture appearance of the obstacles will certainly not be an issue for the propose obstacle detection and avoidance system.
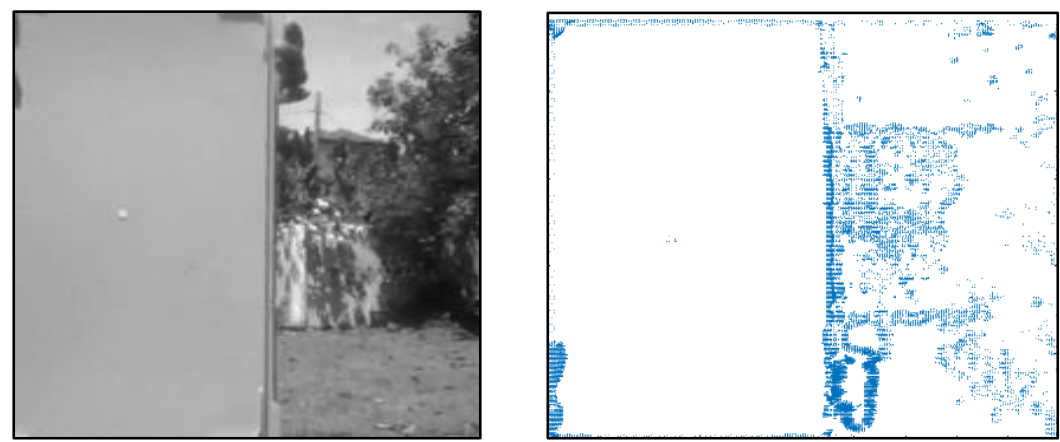

(a)
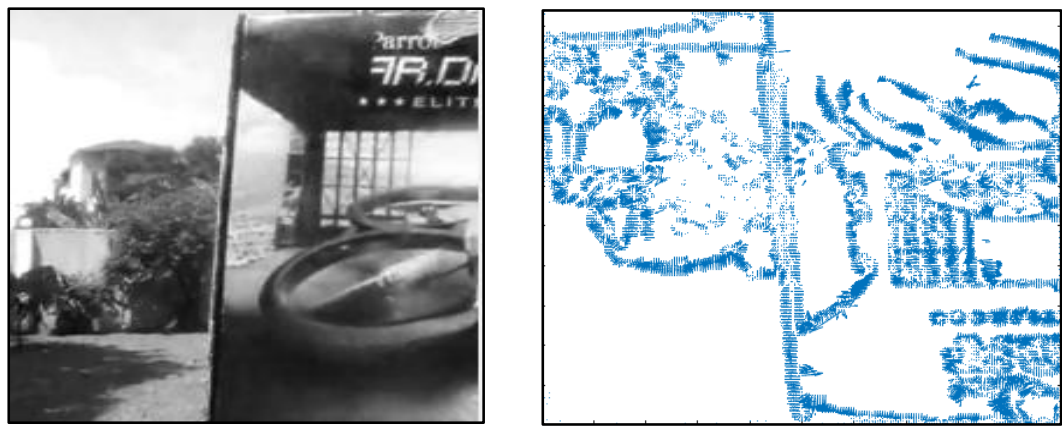

(b)

Fig. 11 - Unpredictable obstacle appearance situation. Left figure and right figure represent the actual image frame of the sudden obstacle appearance and flow field vector generated, respectively. (a) Texture-less obstacle appearance; (b) Textured obstacle appearance

Most of the failures from the obstacle cases are due to the delay of the captured image frame sequence by the camera sensor. The streaming live video from the camera sensor suffers from the streaming latency of about $400 \mathrm{~ms}$ and it is inevitable. Apart from the streaming latency of the camera sensor, the latency is added with the execution of the sudden obstacle appearance algorithm for every image frame. As a consequence, when the obstacle is moving fast in front of the $\mathrm{UAV}$, the camera sensor will not be able to capture the correct image frame sequence concerning the movement of the 
obstacle. When the captured image frames sequence is incorrect, the flow field vectors and the direction of the incoming obstacle will be mistakenly computed by the proposed sudden obstacle appearance algorithm. An example of this matter is when one of the image frames from the image frame sequence does not contain any trace of the introduced obstacle as shown in Figure 12. Most of the vision-based obstacle detection system cannot handle this type of situation due to the inability of the vision-based sensor to determine the depth of the environment [10, 14, 25].
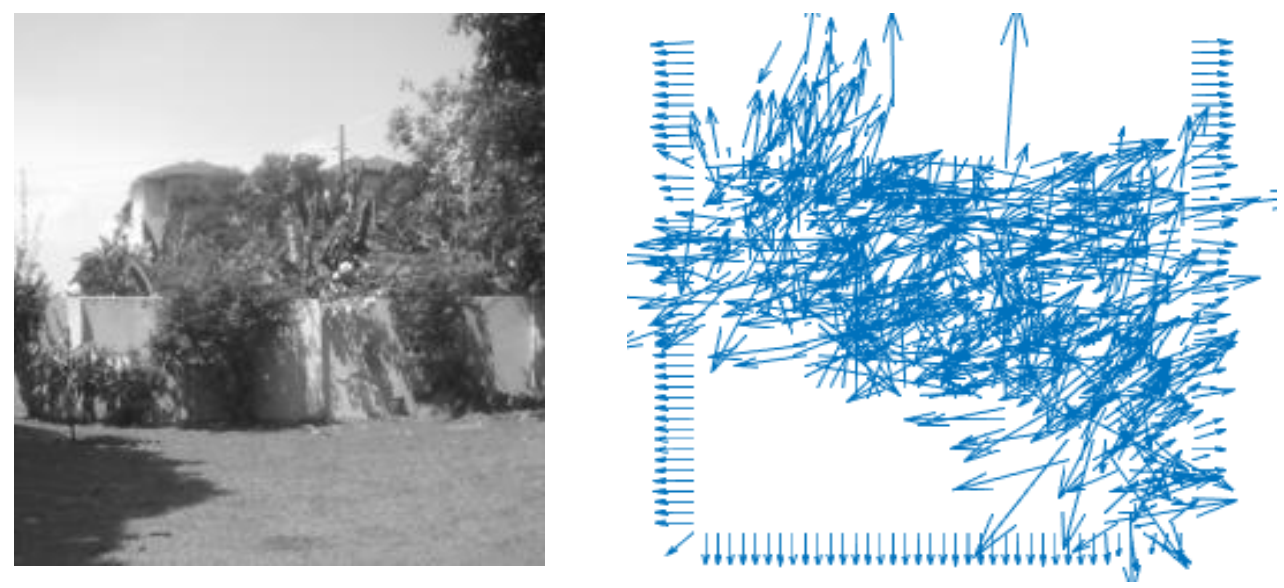

Fig. 12 - Obstacle does not appear in the image frame sequence

\section{Conclusion}

This paper presents research work on the obstacle detection system for the UAV. The aim of this research project is to develop a robust autonomous obstacle detection system for small-sized UAV using multiple and different sensor-based integrations to tackle sudden appearance of the obstacle in front of the UAV. Previous works on this field are bounded by the major constraints of the UAV platform, which is the payload capacity and physical size constraints. There are two types of obstacle detection based, which is vision-based sensor and range-based sensor. These two obstacle detection based types have their own advantages and disadvantages. Due to the mentioned constraints, most of the previous obstacle detection system will only consist of one obstacle detection based. The proposed system solved these obstacle detection difficulties by merging both sensor-based together into a single obstacle detection system. In order to tackle the problem of the sudden appearance of the obstacle, the motion detection method alongside the output from LIDAR Lie v3 is used. The direction of the incoming obstacle is examined through the direction of the flow field vector in the image frame sequence captured by the camera sensor. The results of the experiments indicate that the proposed obstacle detection system is able to handle sudden appearance of obstacle situation with a high degree of success rate. It is found that, the proposed obstacle detection system is also able to create the safe avoidance path even though the texture of the obstacle cannot be detected. The contribution for this work is mainly on mainly on analyzes the flow field vectors pattern to estimate the direction of the incoming obstacle in front of the UAV platform. For future work, the method should be applied as a stand alone detection without interference from ground. This is possible by incorporating the detection algorithm using OpenCv from Python or $\mathrm{C}++$ with the help of powerful microcontroller.

\section{Acknowledgement}

This research was supported by University Tun Hussein Onn Malaysia (UTHM) through Tier 1 (H920).

\section{References}

[1] L. Torrero, A. Molino, T. E. Spa, D. Giordan, I. National, and A. Manconi, "The Use of Micro-UAV to Monitor Active Landslide Scenarios," in Engineering Geology for Society and Territory - Volume 5., 2014, no. September, pp. 701-704, doi: 10.1007/978-3-319-09048-1

[2] C. Deng, S. Wang, Z. Huang, Z. Tan, and J. Liu, "Unmanned aerial vehicles for power line inspection: A cooperative way in platforms and communications," J. Commun., vol. 9, no. 9, pp. 687-692, 2014, doi: $10.12720 / \mathrm{jcm} \cdot 9.9 .687-692$

[3] C. Eschmann, C.-M. Kuo, and C. Boller, "Unmanned Aircraft Systems for Remote Building Inspection and Monitoring," Proc. 6th Eur. Work. Struct. Heal. Monit., vol. 2, pp. 1-8, 2012

[4] J. Scherer et al., "An Autonomous Multi-UAV System for Search and Rescue," Proc. First Work. Micro Aer. Veh. Networks, Syst. Appl. Civ. Use - DroNet'15, pp. 33-38, 2015, doi: 10.1145/2750675.2750683

[5] P. Rudol and P. Doherty, "Human Body Detection and Geolocalization for UAV Search and Rescue Missions Using Color and Thermal Imagery .," in IEEE aerospace conference, 2008, pp. 1-8 
[6] D. Erdos, A. Erdos, and S. E. Watkins, "An experimental UAV system for search and rescue challenge," IEEE Aerosp. Electron. Syst. Mag., vol. 28, no. 5, pp. 32-37, 2013, doi: 10.1109/MAES.2013.6516147

[7] Payal, Akashdeep, and C. R. Singh, "A Summarization of Collision Avoidance Techniques for Autonomous Navigation of UAV," in International Conference on Unmanned Aerial System in Geomatics, 2020, vol. 51, pp. 393-401, doi: 10.1007/978-3-030-37393-1_23

[8] J. C. Zufferey and D. Floreano, "Fly-inspired visual steering of an ultralight indoor aircraft," IEEE Trans. Robot., vol. 22, no. 1, pp. 137-146, 2006, doi: 10.1109/TRO.2005.858857

[9] D. Yoo, D. Won, and M. Tahk, "Optical Flow Based Collision Avoidance of Multi-Rotor UAVs in Urban Environments," Int. J. Aeronaut. Sp. Sci., vol. 12, no. 3, pp. 252-259, 2011, doi: 10.5139/IJASS.2011.12.3.252

[10] A. Al-Kaff, F. García, D. Martín, A. de la Escalera, and J. M. Armingol, "Obstacle detection and avoidance system based on monocular camera and size expansion algorithm for UAVs," Sensors (Switzerland), vol. 17, no. 5, p. 1061, 2017, doi: 10.3390/s17051061

[11] A. H. Al-kaff, "Vision-Based Navigation System for Unmanned Aerial Vehicles," 2017

[12] R. R. Nhair and T. A. Al-Assadi, "Vision-Based Obstacle Avoidance for Small Drone using Monocular Camera," IOP Conf. Ser. Mater. Sci. Eng., vol. 928, no. 3, 2020, doi: 10.1088/1757-899X/928/3/032048

[13] X. She, D. Huang, C. Song, N. Qin, and T. Zhou, "Multi-obstacle detection based on monocular vision for UAV," Proc. 16th IEEE Conf. Ind. Electron. Appl. ICIEA 2021, pp. 1067-1072, 2021, doi: 10.1109/ICIEA51954.2021.9516384

[14] T. Mori and S. Scherer, "First results in detecting and avoiding frontal obstacles from a monocular camera for micro unmanned aerial vehicles," Proc. - IEEE Int. Conf. Robot. Autom., pp. 1750-1757, 2013, doi: 10.1109/ICRA.2013.6630807

[15] W. L. Leong et al., "Vision-Based Sense and Avoid with Monocular Vision and Real-Time Object Detection for UAVs," 2021 Int. Conf. Unmanned Aircr. Syst. ICUAS 2021, pp. 1345-1354, 2021, doi: 10.1109/ICUAS51884.2021.9476746

[16] A. Carrio, J. Tordesillas, S. Vemprala, S. Saripalli, P. Campoy, and J. P. How, "Onboard Detection and Localization of Drones Using Depth Maps," IEEE Access, vol. 8, pp. 30480-30490, 2020, doi: 10.1109/ACCESS.2020.2971938

[17] S. Majumder, R. Shankar, and M. S. Prasad, "Obstacle size and proximity detection using stereo images for agile aerial robots," 2nd Int. Conf. Signal Process. Integr. Networks, SPIN 2015, pp. 437-442, 2015, doi: 10.1109/SPIN.2015.7095261

[18] M. Whalley, M. Takahashi, G. J. Schulein, and C. Goerzen, "Field-testing of a helicopter UAV obstacle field navigation and landing [Online]. Available: http://armyscienceconference.com/manuscripts/E/EP-008.pdf

[19] J. Kim, S. Song, S. Kim, and J. Suk, "Collision Avoidance System for Agricultural Unmanned Helicopter using LIDAR Sensor," 2014

[20] A. Bachrach, R. He, and N. Roy, "Autonomous Flight in Unknown Indoor Environments," Int. J. Micro Air Veh., vol. 1, no. 4, pp. 217-228, 2009, doi: 10.1260/175682909790291492

[21] Z. Šilar and M. Dobrovolný, "Comparison of two optical flow estimation methods using Matlab,” Int. Conf. Appl. Electron., no. 1, pp. 1-4, 2011

[22] D. Patel and U. Saurabh, "Optical Flow Measurement using Lucas kanade Method," Int. J. Comput. Appl., vol. 61, no. 10, pp. 6-10, 2013

[23] B. Y. W. E. Green and P. Y. Oh, "Optic-Flow-Based Collision Avoidance," IEEE Robotics \& Automation Magazine, pp. 96-103, 2008

[24] S. Zingg, D. Scaramuzza, S. Weiss, and R. Siegwart, "MAV navigation through indoor corridors using optical flow," in Proceedings - IEEE International Conference on Robotics and Automation, 2010, pp. 3361-3368, doi: 10.1109/ROBOT.2010.5509777

[25] W. Aguilar, V. Casaliglla, and J. Pólit, "Obstacle Avoidance Based-Visual Navigation for Micro Aerial Vehicles," Electronics, vol. 6, no. 1, p. 10, 2017, doi: 10.3390/electronics6010010 